\title{
Anti-obesity effect of sea buckthorn (Hippohae rhamnoides L) on diet induced obese C57BL/6N mice
}

\author{
Y. S. Cha ${ }^{1}$, H. J. Moon ${ }^{1}$, P. B. Tirupathi Pichiah ${ }^{1}$ and S. H. Oh ${ }^{2}$ \\ ${ }^{1}$ Department Dept. of Food Science \& Human Nutrition, Chonbuk National University, South Korea and \\ ${ }^{2}$ Dept. of Food and Biotechnology, Woosuk University, South Korea
}

\begin{abstract}
Hippohae rhamnoides L (SL), also known as sea buckthorn, is a deciduous shrub species belonging to the genus Hippophae $e^{(1)}$. It is a rich source of many phenolic compounds, which makes it a potential therapeutic agent for treating diverse disease and disorders ${ }^{(2)}$. In the current study we examined the effect of ethanolic extract of sea buckthorn (SL) against diet induced obesity in a rodent model. Thirty two C57BL/6N mice were randomly divided into 4 dietary groups $(n=8)$, normal diet (ND), high fat diet (HD), high fat diet plus SL ethanolic extract oral supplementation $500 \mathrm{mg} / \mathrm{kg}$ bodyweight (SL1), and high fat diet plus SL ethanolic extract oral supplementation $1000 \mathrm{mg} / \mathrm{kg}$ bodyweight (SL2). After 12 weeks of intervention the animals were sacrificed, blood and tissue samples were collected to examine lipid profiles, leptin and insulin concentrations and to evaluate the expression levels of genes involved in lipid metabolism like acetyl-CoA carboxylase (ACC), peroxisome proliferator-activated receptor alpha (PPAR- $\alpha$ ), peroxisome proliferator-activated receptor gamma $($ PPAR- $\gamma)$ and carnitine palmitoyltransferase I $($ CPT-1). Oral administration of SL ethanol extract significantly $(P<0.05)$ reduced feed intake, body weight gain, epididymal fat weight, hepatic triglyceride and cholesterol and serum leptin level in the SL groups comparing with the HD group. Also there was a significant decrease in serum total cholesterol (TC), triglyceride, HDL-cholesterol (HDL-c) and the ratio between HDL-c and TC concentrations (Table).
\end{abstract}

\begin{tabular}{|c|c|c|c|c|}
\hline Groups & ND & HD & SL1 & SL2 \\
\hline Triglyceride & $109.36 \pm 28.33$ & $127.29 \pm 31.45$ & $144.56 \pm 26.49$ & $141.55 \pm 36.54$ \\
\hline Total cholesterol & $88.19 \pm 7.59^{\mathrm{d}}$ & $169.32 \pm 18.54^{\mathrm{a}}$ & $152.90 \pm 10.66^{\mathrm{b}}$ & $129.71 \pm 17.84^{\mathrm{c}}$ \\
\hline HDL-cholesterol & $61.94 \pm 4.03^{c}$ & $105.53 \pm 23.63^{\mathrm{a}}$ & $89.87 \pm 15.82^{\mathrm{ab}}$ & $83.97 \pm 13.75^{\mathrm{b}}$ \\
\hline HDL-c/ TC (\%) & $70.55 \pm 6.06$ & $62.28 \pm 11.69$ & $59.07 \pm 11.22$ & $65.03 \pm 8.49$ \\
\hline
\end{tabular}

The expression of genes involved in fatty acid oxidation namely PPAR alpha and CPT-1; the hypoglycemic effect producing gene PPAR-gamma level was also increased by SL, whereas the level of ACC, the gene involved in fatty acid synthesis was reduced (all $P<0.05$ ).

Our results showed that SL is effective against preventing weight gain, accumulation of fat in liver, it also reduces feed intake, adipose tissue mass, hepatic lipid profile. These properties make SL an effective candidate in management of obesity and related disorders.

This work was supported by grant No. 1101000258 from Korea Food Research Institute.

1. Rousi A (1971) Annals Botany Fennici 8, 177-227.

2. Xu Mingyu (1991) Journal of Water and Soil Conservation of China 5, 38. 